\title{
Heterotic string conformal field theory and $A-D-E$ singularities
}

\author{
Edward Witten \\ California Institute of Technology, Pasadena CA 91125 USA, and \\ CIT-USC Center For Theoretical Physics \\ E-mail: iwittendtheory.càitech.edui
}

Abstract: We analyze the behavior of the heterotic string near an $A-D-E$ singularity without small instantons. This problem is governed by a strongly coupled worldsheet conformal field theory, which, by a combination of $\mathcal{O}\left(\alpha^{\prime}\right)$ corrections and worldsheet instantons, smooths out the singularities present in the classical geometry.

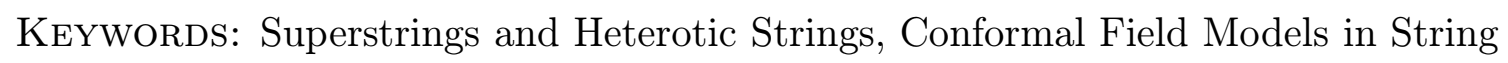
Thêöry:

${ }^{*}$ On leave of absence from Institute for Advanced Study, Olden Lane, Princeton NJ 08540. 


\section{Contents}

ii. Introduction i:

2. Analysis of the moduli space

'2.1' Supergravity analysis

2.-1. The $\mathcal{O}\left(\alpha^{\prime}\right)$ correction

Exact metric

1".-1" Linear sigma model approach

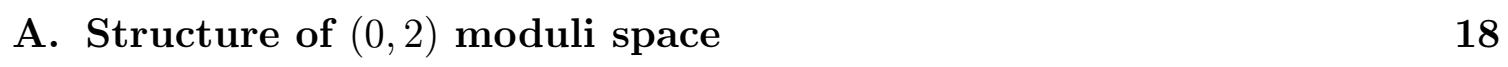

\section{Introduction}

String compactifications with $\mathcal{N}=2$ supersymmetry in four dimensions - and more generally, with eight unbroken supercharges in various dimensions - have been much studied. They are extremely rich in their behavior, yet sufficiently constrained to be analyzed in detail.

The low energy supergravity obtained in $\mathcal{N}=2$ compactification to four dimensions has scalar fields in both vector multiplets and hypermultiplets. The moduli space of vacua (endowed with the metric that appears in the low energy effective action) is [i] li locally a product of a vector multiplet moduli space and a hypermultiplet moduli space.

A prototype of such a compactification is the heterotic string on $\mathrm{K} 3 \times \mathbb{T}^{2}$, which

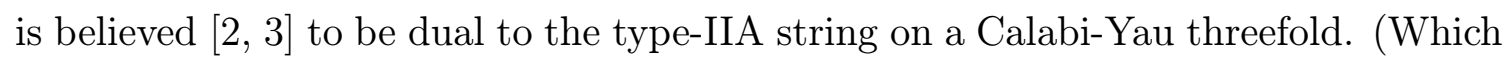
threefold arises here depends on the heterotic string gauge bundle.) In compactification on $\mathrm{K} 3 \times \mathbb{T}^{2}$, the heterotic string dilaton is in a vector multiplet, and the type-IIA dilaton is in a hypermultiplet.

As a result, the vector multiplet moduli space is independent of the type-IIA string coupling, and the hypermultiplet moduli space is independent of the heterotic string coupling. Hence the vector multiplet moduli space can be determined, in principle, from type-IIA conformal field theory, and likewise the hypermultiplet moduli space can be determined, in principle, from heterotic string conformal field theory. This viewpoint has been much exploited for understanding the vector multiplet moduli space [2i, 'Ain. The hypermultiplet moduli space has also been much studied, for

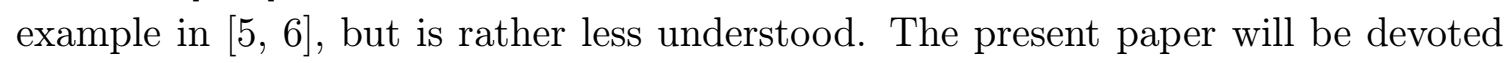
to some issues on the hypermultiplet side, from the standpoint of heterotic string conformal field theory. 
Since conformal field theory on $\mathrm{K} 3 \times \mathbb{T}^{2}$ is the product of conformal field theory on $\mathrm{K} 3$ with (free) conformal field theory on $\mathbb{T}^{2}$, the essential issues will involve the K3 conformal field theory. Thus, our problem will be to study the hypermultiplet moduli space in compactification of the heterotic string on K3. For understanding the hypermultiplets, it does not matter much if one considers K3 compactification to six dimensions or $\mathrm{K} 3 \times \mathbb{T}^{2}$ compactification to four dimensions.

The claim that the moduli spaces can be computed from conformal field theory is subject to an important caveat: the moduli spaces have singularities, which sometimes reflect nonperturbative physics — like the massless hypermultiplet near a type-II conifold [i]1. Thus, one can in principle compute the vector multiplet and hypermultiplet moduli spaces using the appropriate conformal field theories, but one may not be able to understand them.

An important example is the small instanton singularity of the heterotic string. The classical supergravity solution for a small instanton [i8i] shows a blowup of the dilaton near the core of a small instanton, so one must expect a nonperturbative phenomenon to occur as an instanton shrinks to zero size. The phenomenon in question is the appearance of a nonperturbative gauge symmetry [9.9]. Despite its importance in heterotic string dynamics, the small instanton singularity is in the following sense not a good illustration of the role of heterotic string conformal field theory. The instanton moduli space, as computed in classical field theory, has a small instanton singularity, which is uncorrected in going to conformal field theory, ${ }^{1}$ and is interpreted nonperturbatively in terms of enhanced gauge symmetry. Thus, heterotic string conformal field theory (as opposed to supergravity) does not play an important role in generating the singularity. The interpretation of the singularity is also out of reach of conformal field theory. In the present paper, we will analyze an example in which heterotic string conformal field theory does play a central role in controlling the behavior near a classical singularity.

A hint about where to look comes from the classical equation of motion for the dilaton $\phi$, which reads schematically

$$
\Delta^{2} \phi=\operatorname{tr} F_{i j} F^{i j}-\operatorname{tr} R_{i j} R^{i j}
$$

Here $\Delta^{2}$ is the laplacean, $F_{i j}$ is the Yang-Mills curvature of the gauge bundle, and $R_{i j}$ is the Riemann tensor (regarded as a two-form valued in the Lie algebra of the orthogonal group). The crucial point is the relative minus sign between the two terms on the right hand side of this equation. If $F$ is large with $R$ zero, one is driven to strong coupling (as is familiar from the small instanton solution [i] ] , while if $R$ is

\footnotetext{
${ }^{1} \mathrm{~A}$ proof that for $k$-instanton configurations on $\mathbb{R}^{4}$, the classical instanton moduli space coincides with the type-I instanton moduli space can be found in [1 $10_{1}^{\prime}$, section 2.3]. Via heterotic - type-I duality, the same is therefore also true for the heterotic string.
} 
large with $F$ zero, one is driven to weak coupling. So a singularity with large $R$ but zero $F$ should not lead to nonperturbative physics, and should be understandable in the framework of conformal field theory.

We want a singularity that is at finite distance on the moduli space, so we will concentrate on the $A-D-E$ singularities. $A-D-E$ singularities with small instantons have been much studied and give interesting nonperturbative behavior [i] will omit the small instantons so as to get an example governed by conformal field theory. Near the singularity, one can replace the ambient K3 manifold by an ALE space (which is asymptotic to $\mathbb{R}^{4} / \Gamma$ for some finite group $\Gamma ; \Gamma$ depends on the choice of an $A-D-E$ singularity). Since there are no small instantons, we can set $F=0$. So we will study the heterotic string on an ALE space with $F=0$, in the conformal field theory limit. ${ }^{2}$

The conformal field theory moduli space $\mathcal{M}$ for the heterotic string on such a manifold is a hyper-Kähler manifold of dimension $4 r$, where $r$ is the rank of the $A$ - $D$ $E$ group in question. That $\mathcal{M}$ is hyper-Kähler requires some explanation. In general, the hypermultiplet moduli space in a globally supersymmetric theory with eight unbroken supercharges is a hyper-Kähler manifold, but in the presence of gravity it is instead a quaternionic manifold. Focussing on the behavior near a singularity has the effect of decoupling gravity, and that is why $\mathcal{M}$ is hyper-Kähler.

We will focus on the simplest case of $A_{1}=\mathrm{SU}(2)$, so that $r=1$ and $\mathcal{M}$ has real dimension four. In this case, we will in section 2 analyze the structure of $\mathcal{M}$ in the following three steps:

1. In supergravity, one can compute directly that $\mathcal{M}=\left(\mathbb{R}^{3} \times S^{1}\right) / \mathbb{Z}_{2}$, where the $\mathbb{Z}_{2}$ acts by multiplication by -1 on both factors.

2. Going to conformal field theory, there is an $\mathcal{O}\left(\alpha^{\prime}\right)$ correction with the following structure. The correction is singular at the origin (the $\mathbb{Z}_{2}$ fixed point) in $\mathbb{R}^{3}$. Let $\widetilde{\mathbb{R}}^{3}$ be $\mathbb{R}^{3}$ with the origin deleted. Then the $\mathcal{O}\left(\alpha^{\prime}\right)$ correction has the effect of replacing $\widetilde{\mathbb{R}}^{3} \times S^{1}$ by a twisted $S^{1}$ bundle over $\widetilde{\mathbb{R}}^{3}$ (which must then be divided by $\mathbb{Z}_{2}$ to get the moduli space). Since $\widetilde{\mathbb{R}}^{3}$ is homotopic to $S^{2}$, such bundles are classified by an integer-valued first Chern class. In this case, the integer is equal to -4 .

3. There are no further worldsheet perturbative corrections to the metric of $\mathcal{M}$; that is, there are no corrections of order $\left(\alpha^{\prime}\right)^{s}$ with $s>1$. However [1] 6 , there are worldsheet instanton corrections to $\mathcal{M}$. These corrections will preserve the

\footnotetext{
${ }^{2}$ Because we work at string tree level, we will not see the fluctuations around $F=0$, which would (in string loops) distinguish the $E_{8} \times E_{8}$ and $\operatorname{Spin}(32) / \mathbb{Z}_{2}$ heterotic strings. Thus, the two heterotic string theories, compactified on an ALE space without small instantons, have the same moduli space. Note that in the case of $\operatorname{Spin}(32) / \mathbb{Z}_{2}$, since we have taken the gauge field to be trivial, we are considering the case of a gauge bundle with vector structure.
} 
hyper-Kähler structure of $\mathcal{M}$ as well as an $\mathrm{SO}(3)$ action on $\mathcal{M}$ that rotates the complex structures and whose generic orbits are three-dimensional. Moreover, the worldsheet instanton corrections vanish at infinity (on $\mathbb{R}^{3}$ ), and do not modify the asymptotic behavior of $\mathcal{M}$ as found in step 2 above. Four-dimensional hyper-Kähler manifolds with these properties have been analyzed [I] [1] an $\mathcal{M}$ either has a singularity that would be difficult to interpret in conformal field theory, or is a unique, smooth, complete hyper-Kähler four-manifold $\mathcal{M}_{A H}$ described in [i] was identified by Atiyah and Hitchin as the moduli space of BPS dimonopoles in three dimensions.

In sections 12.31 and 2.4 , we give the best arguments we can for why the moduli space should be nonsingular. Among other things, we argue by a simple linear sigma model construction that the $(0,2)$ conformal field theory describing the heterotic string on a Calabi-Yau manifold $Y$ of any complex dimension $n$ never develops a singularity when $Y$ develops an isolated hypersurface singularity near which the gauge fields are trivial.

Steps 1 to 3 above are in precise parallel with similar steps that were used in the determination of the moduli space of vacua for minimal SU(2) supersymmetric gauge theory in three dimensions with eight supercharges [1] consider the theory of the $\mathrm{SU}(2)$ vector multiplet only, without additional charged fields.) In that case (after dualizing the photon to convert the vector multiplet that parametrizes the Coulomb branch of the theory to a hypermultiplet), we have the following statements, in close parallel to the above: (1) the classical moduli space of vacua is $\left(\mathbb{R}^{3} \times S^{1}\right) / \mathbb{Z}_{2} ;(2)$ there is a one-loop correction that replaces the product $\mathbb{R}^{3} \times S^{1}$ by a twisted fiber bundle; (3) there are instanton corrections that turn the moduli space into $\mathcal{M}_{A H}$.

This analogy suggests a generalization of our result to other $A-D-E$ singularities. Consider the heterotic string at a singularity of type $G$ without small instantons, where $G$ is a group of $A, D$, or $E$ type. The conjecture is that the hypermultiplet moduli space for the heterotic string near such a singularity is the moduli space of vacua of a minimal supersymmetric gauge theory in three dimensions with eight supercharges and gauge group $G$.

After submitting to hep-th the original version of this paper, I became aware that Sen has treated the $A_{n}$ case of this problem, by considering the heterotic string on a multi-Taub-NUT spacetime [20-0]. (The multi-Taub-NUT example is relevant because it can develop an $A_{n}$ singularity.) In this approach, the connection to BPS multimonopoles of $\mathrm{SU}(2)$ gauge theory is made by going very close to the self-dual radius of the heterotic string on a circle, where an enhanced $\mathrm{SU}(2)$ gauge symmetry appears. See also [2]-1, for a prior discussion of the relation of $H$-monopoles to BPS monopoles. 


\section{Analysis of the moduli space}

As was explained in the introduction, we will here analyze the behavior of the heterotic string at an $A_{1}$ singularity without small instantons. An $A_{1}$ singularity is simply a quotient singularity of the form $\mathbb{R}^{4} / \mathbb{Z}_{2}$, where the generator of $\mathbb{Z}_{2}$ acts on $\mathbb{R}^{4}$ by multiplication by -1 . The analysis will come in the three stages described in the introduction: (1) supergravity; (2) incorporation of an $\mathcal{O}\left(\alpha^{\prime}\right)$ correction; (3) exact description using worldsheet instantons.

\subsection{Supergravity analysis}

In string theory, when one divides $\mathbb{R}^{4}$ by $\mathbb{Z}_{2}$ to form the orbifold $\mathbb{R}^{4} / \mathbb{Z}_{2}$, one must pick the sign of the action of $\mathbb{Z}_{2}$ on fermions. Either choice leaves half of the supersymmetry unbroken and determines a distinguished orientation on $\mathbb{R}^{4} / \mathbb{Z}_{2}$. With this distinguished orientation, $\mathbb{R}^{4} / \mathbb{Z}_{2}$ is a flat hyper-Kähler manifold with an isolated orbifold singularity. As a hyper-Kähler manifold, $X$ has a two-sphere of complex structures. If $I, J$, and $K$ are the quaternion generators on $\mathbb{R}^{4}$, then the general complex structure is $w_{1} I+w_{2} J+w_{3} K$, where $w_{1}^{2}+w_{2}^{2}+w_{3}^{2}=1$. The symmetry group of $X=\mathbb{R}^{4} / \mathbb{Z}_{2}$ is $\mathrm{SO}(4)=\mathrm{SU}(2)_{L} \times \mathrm{SU}(2)_{R}$ where (with a suitable choice of orientation) $I, J$, and $K$ are invariant under $\mathrm{SU}(2)_{L}$ and transform with spin one under $\mathrm{SU}(2)_{R}$. Hence a choice of $\vec{w}$ breaks $\mathrm{SU}(2)_{L} \times \mathrm{SU}(2)_{R}$ to $\mathrm{SU}(2)_{L} \times \mathrm{U}(1)_{R}$.

If one picks a particular $\vec{w}$, that is a particular complex structure on $X$, then one can "blow up" the orbifold singularity of $X$ in that complex structure to make a smooth ALE hyper-Kähler manifold, the Eguchi-Hansen space $X^{\prime}$ [를ㄹ. In such a blowup, there is a projection $X^{\prime} \rightarrow X$ which is generically one-to-one and is holomorphic in the complex structure specified by $\vec{w}$. The blowup of $X$ to make $X^{\prime}$ is completely determined by the choice of $\vec{w}$ and the area $\alpha$ of the exceptional divisor produced in the blowup. We can combine $\vec{w}$ and $\alpha$ to a three-vector $\vec{m}$ whose direction is the unit vector $\vec{w}$ and whose magnitude determines $\alpha$. (A convenient way to do this is described below.) $\vec{m}$ is a completely arbitrary element of $\mathbb{R}^{3}$, so at first sight it seems that the moduli space of hyper-Kähler blowups of $X$ (modulo diffeomorphisms that are trivial at infinity) is a copy of $\mathbb{R}^{3}$.

However, the complex structure $\vec{w}$ with respect to which the blowup is made is not quite uniquely determined. The projection $X^{\prime} \rightarrow X$ is holomorphic with respect to both the complex structure determined by $\vec{w}$, and the "opposite" (or complex conjugate) complex structure determined by $-\vec{w}$. Hence, we should identify $\vec{m}$ with $-\vec{m}$, and the moduli space of blowups is actually $\mathbb{R}^{3} / \mathbb{Z}_{2}$.

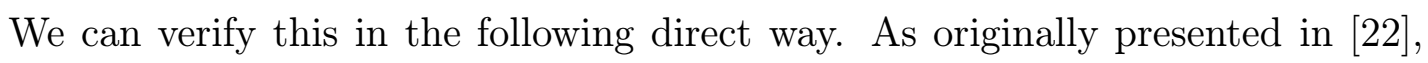
the metric of $X^{\prime}$ reads

$$
d s^{2}=f(r)^{2} d r^{2}+r^{2}\left(\sigma_{1}^{2}+\sigma_{2}^{2}\right)+r^{2} g^{2} \sigma_{3}^{2},
$$


with

$$
g=f^{-1}=\sqrt{1-\left(\frac{a}{r}\right)^{4}}
$$

here $\sigma_{1}, \sigma_{2}$, and $\sigma_{3}$ are the left-invariant one-forms on $S^{3} \cong \mathrm{SU}(2)$, and $a$ is a constant that can be identfied with $|\vec{m}|$. Note that the distinguished role of $\sigma_{3}$ in the formula breaks the $\mathrm{SU}(2)_{L} \times \mathrm{SU}(2)_{R}$ symmetry of the $\mathrm{SU}(2)$ manifold to $\mathrm{SU}(2)_{L} \times \mathrm{U}(1)_{R}$, as expected. We can readily generalize ( $\left(2 . \bar{l}_{1}^{\prime}\right)$ to a more general blowup in which $\sigma_{3}$ is replaced by a more general linear combination of the $\sigma$ 's. We introduce an arbitrary unit vector $\vec{w}$ and generalize $\left(\underline{2}, \overline{1} \overline{1}_{1}^{\prime}\right)$ to

$$
d s^{2}=f(r)^{2} d r^{2}+r^{2}\left(\sum_{i=1}^{3} \sigma_{i}^{2}-(\vec{w} \cdot \vec{\sigma})^{2}\right)+r^{2} g(r)^{2}(\vec{w} \cdot \vec{\sigma})^{2} .
$$

This is invariant under $\vec{w} \rightarrow-\vec{w}$, so we should consider $\vec{w}$, and hence also $\vec{m}=a \vec{w}$, to be defined only up to sign.

The exceptional divisor $S$ produced in the blowup is the two-sphere at $r=a$ (where the coefficient of $\sigma_{3}$ in $\left(\underline{2}, \overline{1}_{1}^{\prime}\right)$ vanishes and the $S^{3}$ collapses to an $S^{2}$ ). Its area is $\alpha=4 \pi a^{2}$.

Now, let us include the $B$-field. This introduces one more real modulus, which is essentially the period of the $B$-field integrated over $S$ :

$$
\theta=\int_{S} B
$$

Modulo global gauge transformations of the $B$ field, $\theta$ is an angular variable, of period $2 \pi$. At first sight, then, it seems that the supergravity moduli space of $X^{\prime}$ is a product $\mathbb{R}^{3} / \mathbb{Z}_{2} \times S^{1}$, where the first factor allows for the blowup and the second for the theta angle. But $\mathbb{R}^{3} / \mathbb{Z}_{2} \times S^{1}$ is not hyper-Kähler, so inevitably there is a subtlety here. To make sense of $\theta$ as a number, we need an orientation of $S$. While $X^{\prime}$ has a natural orientation, $S$ does not. A choice of complex structure on $X^{\prime}$ determines a complex structure and hence an orientation of $S$, but if we reverse the complex structure of $X^{\prime}$, the complex structure and orientation of $S$ will be reversed.

So under $\vec{w} \rightarrow-\vec{w}$, the orientation of $S$ is reversed and $\theta$ is mapped to $-\theta$. The supergravity moduli space of $X^{\prime}$ is thus

$$
\mathcal{M}_{\text {SUGRA }}=\left(\mathbb{R}^{3} \times S^{1}\right) / \mathbb{Z}_{2},
$$

with $\mathbb{Z}_{2}$ acting as -1 on both $\mathbb{R}^{3}$ and $S^{1}$. This carries a natural flat hyper-Kähler metric, with two isolated orbifold singularities.

What about the symmetries of $\mathcal{M}_{\text {SUGRA }}$ ? $\mathrm{SU}(2)_{L}$ acts trivially on $\mathcal{M}_{\text {SUGRA }}$, since it is left unbroken by the blowup, regardless of the choice of $\vec{w}$, and hence acts trivially on $\vec{w}$. But $\mathrm{SU}(2)_{R}$ acts on $\mathcal{M}_{\text {SUGRA }}=\left(\mathbb{R}^{3} \times S^{1}\right) / \mathbb{Z}_{2}$ as the group of rotations of $\mathbb{R}^{3}$. It rotates the complex structures of $\mathcal{M}_{\text {SUGRA }}$ just as it did to the 
original orbifold $X$. The double cover $\mathbb{R}^{3} \times S^{1}$ also has a U(1) symmetry, which we will call $\mathrm{U}(1)_{A}$, that rotates the $S^{1}$ factor, adding a constant to the period of the $B$-field. $\mathrm{SU}(2)_{R}$, because it originates in the symmetries of the original orbifold $X$, is an exact symmetry of the conformal field theory moduli space, but $\mathrm{U}(1)_{A}$ is broken by worldsheet instantons.

Topology. To compute the $\mathcal{O}\left(\alpha^{\prime}\right)$ correction to the moduli space in section 2.2, , we will need some information about the topology of $X^{\prime}$, and in more detail about the behavior of $X^{\prime}$ as $\vec{w}$ varies.

For fixed and nonzero $\vec{w}, X^{\prime}$ is the cotangent bundle $T^{*} S$ of the exceptional divisor $S$. This is a standard fact, which we review below. For the present discussion, we will mainly limit ourselves to the case of a unit blowup, $|\vec{w}|=1$; the reason for this is that as long as the area of the exceptional divisor is nonzero, it can be scaled out and does not affect the topology. We will also mostly ignore the discrete identification $\vec{w} \rightarrow-\vec{w}$, and consider $\vec{w}$ to parametrize a two-sphere $W$. At the end of the discussion, one can divide by $\mathbb{Z}_{2}$.

As $\vec{w}$ varies in $W, X^{\prime}$ varies as the fibers of a six-manifold $Y$ that is fibered over $W . Y$ is not a simple product $X^{\prime} \times W$. However, if we restrict ourselves to the exceptional divisor, we do get a simple product $S \times W$. This is ensured by the $\mathrm{SU}(2)_{L} \times \mathrm{SU}(2)_{R}$ group action, with $\mathrm{SU}(2)_{L}$ and $\mathrm{SU}(2)_{R}$ acting, respectively, by rotations of $S$ and $W$. Replacing $S \times W$ by a nontrivial fibration would spoil the $\mathrm{SU}(2)_{L} \times \mathrm{SU}(2)_{R}$ symmetry. Thus $Y$ contains an embedded copy $S \times W$ of $S^{2} \times S^{2}$. The normal bundle $N$ to $S \times W$ in $Y$ is a real two-plane bundle, which we can alternatively regard as a complex line bundle $\mathcal{R}$. A complex line bundle is labeled topologically by its first Chern class. In the present case, the first Chern class of $\mathcal{R}$ takes values in $H^{2}(S \times W ; \mathbb{Z})=\mathbb{Z} \times \mathbb{Z}$, and is determined by a pair of integers which are the components of the first Chern class along $S$ and $W$, respectively. ${ }^{3}$ We will show that these integers are $(2,2)$, a fact that will be used in section 2.22 to determine the $\mathcal{O}\left(\alpha^{\prime}\right)$ correction.

To show this, we will use an alternative description of $X$ and $X^{\prime}$ as a hyperKähler quotients [2 $\left[\begin{array}{l}2 \\ 2\end{array}\right.$ here $A$ and $A^{\prime}$ transform as spin $1 / 2$ of $\mathrm{SU}(2)_{L}$ and $\mathrm{SU}(2)_{R}$, respectively, and in addition $a^{A A^{\prime}}$ has charge 1 with respect to a $\mathrm{U}(1)$ gauge group that we will call $\mathrm{U}(1)_{G}$. The hyper-Kähler moment map condition (for a supersymmetric vacuum after gauging of $\left.\mathrm{U}(1)_{G}\right)$ is

$$
\sum_{A} a^{A A^{\prime}} \bar{a}_{A B^{\prime}}=\vec{w} \cdot \vec{\sigma}^{A^{\prime}}{ }_{B^{\prime}}
$$

with $\vec{\sigma}$ the Pauli $\sigma$-matrices. After imposing this condition and dividing by $\mathrm{U}(1)_{G}$, one gets for $\vec{w}=0$ the orbifold $X=\mathbb{R}^{4} / \mathbb{Z}_{2}$, and for $\vec{w} \neq 0$ the resolution $X^{\prime}$.

\footnotetext{
${ }^{3}$ To interpret these components as integers requires orienting $S$ and $W$. We will not be precise about the orientations, so some of our statements only hold up to sign.
} 
Let explicitly $a^{A A^{\prime}}=u^{A} \delta^{A^{\prime} 1}+v^{A} \delta^{A^{\prime} 2}$. The hyper-Kähler moment map equation is in more detail

$$
\begin{aligned}
\sum_{A}\left(\left|u^{A}\right|^{2}-\left|v^{A}\right|^{2}\right) & =w_{3} \\
\sum_{A} u^{A} \bar{v}_{A} & =w_{1}+i w_{2} .
\end{aligned}
$$

For example, suppose $w_{1}=w_{2}=0, w_{3}=1$. If we set $v^{A}=0$ and divide by $\mathrm{U}(1)_{G}$, we get a copy of $\mathbb{C P}^{1}$ which is the exceptional divisor $S$. Relaxing the condition $v^{A}=0$, the equation $\sum_{A} u^{A} \bar{v}_{A}=0$ shows that $v$ is a cotangent vector to $S=\mathbb{C P}^{1}$, so that $X^{\prime}$ is the cotangent bundle of $S$, as mentioned earlier.

Now we want to describe the normal bundle $N$, or equivalently the complex line bundle $\mathcal{R}$. Since $\mathcal{R}$ is a homogeneous (SU $(2)_{L} \times \mathrm{SU}(2)_{R}$-invariant) line bundle over the homogeneous space $S \times W$, it can be uniquely determined by describing the group action. Picking a point $P$ (such as the point where $w_{1}, w_{2}$, and $u^{2}$ all vanish) in $S \times W, \mathrm{SU}(2)_{L} \times \mathrm{SU}(2)_{R}$ is broken down to $\mathrm{U}(1)_{L} \times \mathrm{U}(1)_{R} \cdot \mathrm{U}(1)_{L} \times \mathrm{U}(1)_{R}$ acts on the fiber of $\mathcal{L}$ at $P$ with some charges, say $(n, m)$, and these are the components of the first Chern class. We will now show that the charges are $(2,2)$.

In fact, $\mathrm{U}(1)_{L}$ is the symmetry under which $u^{1}$ and $v^{1}$ have charge 1 and $u^{2}$ and $v^{2}$ have charge -1 , while $\mathrm{U}(1)_{R}$ is the symmetry that assigns charge 1 to $u^{A}$ and -1 to $v^{A}$. The $\mathrm{U}(1)_{G^{-}}$-invariant coordinate on the fiber of $\mathcal{L}$ over $P$ is $q=u^{1} \bar{v}_{2}$, which has charge 2 for both $\mathrm{U}(1)_{L}$ and $\mathrm{U}(1)_{R}$, as promised.

To conclude, we will tie up a detail. Using the description in ( $\left(\overline{2} \cdot \overline{6}^{\prime}\right)$, the identification $\vec{w} \leftrightarrow-\vec{w}$ is not very evident. This identification arises because the transformation $\tau: a^{A A^{\prime}} \leftrightarrow \epsilon^{A B} \epsilon^{A^{\prime} B^{\prime}} \bar{a}_{B B^{\prime}}$ maps $\vec{w} \rightarrow-\vec{w}$, so resolutions of the singularity with equal and opposite $\vec{w}$ are equivalent by the action of $\tau$. Moreover (after imposing ( $(1.6)$ and dividing by $\left.\mathrm{U}(1)_{G}\right), \tau$ acts trivially for $|a| \rightarrow \infty$. The last condition is important, because in describing the moduli of $X^{\prime}$, we classify the resolutions up to diffeomorphisms that are trivial near infinity; all of the deformations with the same $|\vec{w}|$ are in fact equivalent by an $\mathrm{SU}(2)_{R}$ rotation which acts nontrivially at infinity.

\subsection{The $\mathcal{O}\left(\alpha^{\prime}\right)$ correction}

In this subsection, we carry out the second step in analyzing the heterotic string moduli space on the ALE space without small instantons. This is to analyze the corrections to the moduli space coming from worldsheet perturbation theory. We will find that the perturbative correction to the metric is completely determined by an $\mathcal{O}\left(\alpha^{\prime}\right)$ term that can be described in terms of topology.

The supergravity moduli space $\mathcal{M}_{\mathrm{SUGRA}}=\left(\mathbb{R}^{3} \times S^{1}\right) / \mathbb{Z}_{2}$ that we found in (12.5. is an $S^{1}$ bundle over $\mathcal{W}=\mathbb{R}^{3} / \mathbb{Z}_{2}$, where the fibration is described by forgetting $\theta$. This fibration is flat: it is trivial if lifted to $\mathbb{R}^{3}$ (since the double cover of $\mathcal{M}_{\text {SUGRA }}$ is a product $\mathbb{R}^{3} \times S^{1}$ ). For type-II superstrings, something like this is the complete 
answer to all orders in $\alpha^{\prime}$ : the $B$-field periods take values in a torus bundle (or a circle bundle when there is only one period, as in the case that we are studying) that is flat, with discrete monodromies (which are associated with the mapping class group and singularities). For the heterotic string, however, the $B$-field periods take values in a circle or torus bundle $\mathcal{T}$ that is not flat. (In addition, as we will see, the fibration structure breaks down near certain singularities.)

Note that a circle bundle $\mathcal{V}$ is closely related to a complex line bundle $\mathcal{V}^{\prime} ; \mathcal{V}$ is the bundle of unit vectors in $\mathcal{V}^{\prime}$. We will write $c_{1}(\mathcal{V})$ as an abbreviation for $c_{1}\left(\mathcal{V}^{\prime}\right)$. A torus bundle $\mathcal{T}$ is similarly related to a bundle whose fiber is $\mathbb{C}^{r}$, with $r$ the rank of the torus.

Here are two related approaches to analyzing the torus bundle $\mathcal{T}$ :

1. For type-II, the field strength of $B$ is $H=d B$, and the Bianchi identity reads

$$
d H=0 .
$$

For the heterotic strings, there are additional Chern-Simons terms in the definition of $H$, and the Bianchi identity reads $d H=(\operatorname{tr} F \wedge R-\operatorname{tr} R \wedge R) / 4 \pi$, where $F$ and $R$ are the Yang-Mills and Riemann curvature two-forms. In the present paper, we set $F=0$, so the Bianchi identity (after dividing by $2 \pi$ for convenience since $H / 2 \pi$ has integral periods) is

$$
d\left(\frac{H}{2 \pi}\right)=-\frac{1}{8 \pi^{2}} \operatorname{tr} R \wedge R
$$

The right hand side comes from an $\mathcal{O}\left(\alpha^{\prime}\right)$ correction in the worldsheet theory (though we have set $\alpha^{\prime}=1 / 2 \pi$ in writing the formula). For a given target space $X^{\prime}$ of the heterotic string, $\left(\overline{2}_{-} . \overline{9}_{1}^{\prime}\right)$ has solutions (or the model would be altogether inconsistent). Now suppose that $X^{\prime}$ varies in its moduli space. If there exists a smoothly varying solution $H_{0}$ of $\left(\underline{2}_{-}^{-} . \overline{9}_{1}^{\prime}\right)$, then one can set $H=H_{0}+d B^{\prime}$, where $B^{\prime}$ is an "ordinary two-form gauge field" (whose field strength, for example, obeys conventional Dirac quantization); by taking the periods of $B^{\prime}$ as coordinates, this would trivialize $\mathcal{T}$. The obstruction to trivializing $\mathcal{T}$ is thus the obstruction to picking a smoothly varying $H_{0}$. This gives us a framework for describing $\mathcal{T}$; if the $B$-field has only one period $\int_{S} B$, so $\mathcal{T}$ is a circle bundle, then

$$
\int_{W} c_{1}(\mathcal{T})=\int_{S \times W}\left(-\frac{1}{8 \pi^{2}} \operatorname{tr} R \wedge R\right)
$$

for any two-cycle $W$ in the moduli space. ${ }^{4}$ This formula completely determines $c_{1}(\mathcal{T})$ modulo torsion. In the present case there is only one relevant two-cycle

\footnotetext{
${ }^{4}$ We are over-simplifying a bit. In general, in the above formula, $S$ varies with $W$, and $S \times W$ must be replaced by the total space of a fiber bundle, with fiber $S$ and base $W$.
} 
and no possibility of torsion. There is a natural parallel transport of $H_{0}$ that comes by asking that its change (when the metric of $X^{\prime}$ and hence the right hand side of (2..9.1) varies) be as small as possible; in going around a loop in moduli space, $H_{0}$ does not come back to itself, which is why $\mathcal{T}$ is not flat. Obviously, if $\mathcal{T}$ were flat, then the left hand side of $(2.10 .101)$ would vanish.

2. A related and more precise approach (which, for example, could be used in a more complicated situation to determine the torsion in $\left.c_{1}(\mathcal{T})\right)$ is as follows. Suppose that we want to study the period of $B$ integrated over a two-cycle $S$ in spacetime. One factor in the worldsheet path integral is the coupling $\exp \left(i \int_{S} B\right)$ to the $B$-field; another factor is the pfaffian $\operatorname{Pf}(\mathcal{D})$ of the worldsheet Dirac operator $\mathcal{D}$. The product

$$
\exp \left(i \int_{S} B\right) \cdot \operatorname{Pf}(\mathcal{D})
$$

must be well-defined. The pfaffian $\operatorname{Pf}(\mathcal{D})$ takes values in a "pfaffian line bundle" $\mathcal{L}$. Hence, $\exp \left(i \int_{S} B\right)$ must be a section of $\mathcal{L}^{-1}$. So the period $\int_{S} B$ of the $B$ field does not take values in $\mathbb{R} / 2 \pi \mathbb{Z}$ but in a circle bundle $\mathcal{T}$ which is the bundle of unit vectors in $\mathcal{L}^{-1}$. (This description of $\mathcal{T}$, which is discussed in more detail in $[1] \overline{1} \overline{6}]$, can be reduced to the previous one by using the Quillen formula for the curvature of $\operatorname{Pf}(\mathcal{D})$.)

In our problem of strings on the ALE space $X^{\prime}$, the moduli space of hyperKähler metrics is $\mathbb{R}^{3} / \mathbb{Z}_{2}$. At the origin in $\mathbb{R}^{3} / \mathbb{Z}_{2}, X^{\prime}$ develops a singularity and the $\alpha^{\prime}$ expansion breaks down, as do the definition and interpretation of the $B$-field period. Hence, in analyzing the $\mathcal{O}\left(\alpha^{\prime}\right)$ correction, we will work away from the origin in $\mathbb{R}^{3} / \mathbb{Z}_{2}$. As in the discussion at the end of section '2. 1 ', this means for topological purposes that we can replace $\mathbb{R}^{3} / \mathbb{Z}_{2}$ with the unit sphere $W$ defined by $|\vec{w}|=1 ; W$ should be divided by $\mathbb{Z}_{2}$ at the end of the discussion.

In the particular case that we are looking at, the $B$-field has only one period, namely $\theta=\int_{S} B$ with $S$ the exceptional divisor. This period takes values in a circle bundle $\mathcal{T}$ over $W$; we wish to compute the first Chern class of $\mathcal{T}$. We will do this using the approaches 1 and 2 above:

1'. The characteristic class $-\operatorname{tr} R \wedge R / 8 \pi^{2}$ that appears in the Bianchi identity $\left(\underline{2} \cdot \bar{g}_{1}^{\prime}\right)$ is $-p_{1} / 2$, where $p_{1}$ is the first Pontryagin class. As in section $\underline{2} . \overline{1}_{-}^{\prime}$, let $Y$ be the six-manifold fibered over $W$ with fiber $X^{\prime}$ (the fiber over $w \in W$ being $X^{\prime}$ with moduli determined by $\left.\vec{w}\right)$. (2.2.

$$
\int_{W} c_{1}(\mathcal{T})=-\frac{1}{2} \int_{S \times W} p_{1}\left(T X^{\prime}\right),
$$

with $T X^{\prime}$ the tangent bundle of $X^{\prime}$. (It would not matter if we used the tangent bundle of $Y$ instead.) In section $2 .{ }_{1}^{1}$, we showed that $Y$ is fibered over 
$S \times W$ with normal bundle a two-plane bundle $N$ or equivalently a complex line bundle $\mathcal{R}$. Because $Y$ is contractible to $S \times W$, the class $-p_{1}\left(T X^{\prime}\right) / 2$ is a pullback from $S \times W$. To evaluate it, we note that $T X^{\prime}$ restricted to $S \times W$ is $T S \oplus N$ (where $T S$ is the tangent bundle of $S$ ). As $T S$ is stably trivial, it does not contribute to $p_{1}\left(T X^{\prime}\right)$, which hence receives a contribution only from $N$. In general, one has for any real vector bundle $Q, p_{1}(Q)=\sum_{i} x_{i}^{2}$, where the $x_{i}$ are the roots of the Chern polynomial. For $Q$ a two-plane bundle $N$ that is associated with a complex line bundle $\mathcal{R}$, there is only one root, which is $c_{1}(\mathcal{R})$. We computed this in section $\mathfrak{2}_{-1}^{1}$ to be $2[S]+2[W]$, where the intersection numbers are $[S]^{2}=[W]^{2}=0,[S] \cdot[W]=1$. So $-\int_{S \times W} p_{1} / 2=$ $-(1 / 2) \int_{S \times W} c_{1}(\mathcal{R})^{2}=-(1 / 2)(2[S]+2[W])^{2}=-4$. The first Chern class of the line bundle $\mathcal{T}$ over $W$ is thus -4 .

2'. For the second approach, we take $S$ in $\left(2.1 \overline{1}_{1}\right)$ to be the exceptional divisor, and we must identify the pfaffian line bundle $\mathcal{L}$ as a line bundle over $W . W$ is a two-sphere that is a homogeneous space for $\mathrm{SU}(2)_{R}$, and a given point $w \in W$ is invariant under a subgroup $\mathrm{U}(1)_{R}$ of $\mathrm{SU}(2)_{R}$. The first Chern class of $\mathcal{L}$ is simply the "charge" (or the eigenvalue of the generator) with which $\mathrm{U}(1)_{R}$ acts on the fiber of $\mathcal{L}$ over $w$. But this fiber is simply the top exterior power of the space of zero modes of the worldsheet fermions of the heterotic string, with worldsheet $S$. So the charge of the fiber is the sum of the charges of the zero modes. The heterotic string worldsheet fermions are left-moving gauge fermions $\lambda$, which in our problem have no zero modes since we have taken the gauge fields to vanish, and right-moving spacetime fermions $\psi$, which are spinors on $S$ with values in the tangent bundle to $X^{\prime}$. The only modes of $\psi$ that matter for computing the $\mathrm{U}(1)_{R}$ action on the fiber of $\mathcal{L}$ are the modes that transform nontrivially under $\mathrm{U}(1)_{R}$; these are the modes that are sections of the normal bundle $N$ (to $S$ in $X^{\prime}$ ). As we explained in section $2 . \overline{1}, N$ is the cotangent bundle to $S$, rotated with charge 2 by $\mathrm{U}(1)_{R}$. Holomorphically, the spin bundle of $S$ is the holomorphic bundle $\mathcal{O}(-1) . N$ is the real cotangent bundle of $S$; its complexification splits holomorphically as $\mathcal{O}(-2)^{-2} \oplus \mathcal{O}(2)^{2}$, where the exponent is the $\mathrm{U}(1)_{R}$ charge. Tensoring this with the spin bundle $\mathcal{O}(-1)$, it follows that spinors on $S$ with values in $N$ are the sum of $\mathcal{O}(-3)^{-2}$, with no zero modes, and $\mathcal{O}(1)^{2}$, with two zero modes. Since these two zero modes each have charge 2 , the total $\mathrm{U}(1)$ charge of the zero modes is $2+2=4$. The first Chern class of $\mathcal{L}$ is hence 4 , and so the inverse bundle $\mathcal{T}$, of which the $B$-field period is a section, has first Chern class -4 .

The metric. The reader may be perplexed: our goal was to compute the string perturbation theory corrections to the metric on the moduli space $\mathcal{M}$, and instead we have computed the first Chern class of a line bundle. 
There is, however, a simple relation between the two questions. As noted in section $2 \overline{2} . \overline{1}$, the supergravity moduli space, before dividing by the discrete symmetry $\tau$, has a $\mathrm{U}(1)_{A}$ symmetry that rotates the period of $B$ by $\theta \rightarrow \theta+c$ for any angle $c$. This is also a symmetry of the $\alpha^{\prime}$ expansion, since the zero mode of $B$ decouples in sigma model perturbation theory. The metric on $\mathcal{M}$ computed in sigma model perturbation theory therefore has this $\mathrm{U}(1)$ symmetry. It also, of course, has $\mathrm{SU}(2)_{R}$ symmetry, induced from the geometric symmetries of $X$ that are broken by the blowup. It acts with generic orbits three-dimensional, since the first Chern class at infinity is nonzero. (If indeed $\mathrm{SU}(2)_{R}$ acted only on the base and not the fiber of the fibration at infinity, then the $\mathrm{SU}(2)_{R}$ orbits would give a trivialization of that fibration.)

Hyper-Kähler metrics in four dimensions with this kind of $\mathrm{SU}(2) \times \mathrm{U}(1)$ symmetry have been classified [i] and are completely determined by the topology at infinity. Such metrics are constructed from the Euclidean Taub-NUT space. This space can be obtained by a hyper-Kähler quotient [2.5] and can be explicitly described by the metric

$$
d s^{2}=\frac{1}{4}\left(\frac{1}{|\vec{x}|}+\frac{1}{\lambda^{2}}\right) d \vec{x}^{2}+\frac{1}{4}\left(\frac{1}{|\vec{x}|}+\frac{1}{\lambda^{2}}\right)^{-1}(d \theta+\vec{\omega} \cdot d \vec{x})^{2},
$$

with $\lambda$ a constant and $\vec{\omega}$ the Dirac monopole potential on $\mathbb{R}^{3}$. This manifold is smooth and has the topology of $\mathbb{R}^{4}$; the group $\mathrm{U}(1)_{A}$ of shifts of $\theta$ has a fixed point at the origin. The fixed point means that at the origin, there is no such thing as "the period of the $B$-field". At infinity, the Taub-NUT space looks like an $S^{1}$ bundle over $\mathbb{R}^{3}$ of first Chern class -1 . To get first Chern class $-n$ at infinity, with $n>0$, still over $\mathbb{R}^{3}$, one divides by $\theta \rightarrow \theta+2 \pi / n$, producing a $\mathbb{Z}_{n}$ orbifold singularity at the origin. (First Chern class $+n$ at infinity with $n>0$ is obtained from the same metric with opposite orientation of the fiber.) In our case, $n=4$, and we want the structure at infinity to be that of an $S^{1}$ bundle over $\mathbb{R}^{3} / \mathbb{Z}_{2}$, not $\mathbb{R}^{3}$, so we must divide by an additional $\mathbb{Z}_{2}$. The generator of this $\mathbb{Z}_{2}$ acts on $\theta$ by $\theta \rightarrow-\theta$; this transformation together with the $\mathbb{Z}_{4}$ symmetry $\theta \rightarrow \theta+\pi / 2$ generates a dihedral group $D_{4}$, with eight elements. Thus, the topology of the spacetime is $\mathbb{R}^{4} / D_{4}$, and there is an isolated $D_{4}$ singularity at the origin.

How could this metric be obtained from a detailed calculation, rather than being deduced from the topology as we have done? In supergravity, the metric arises by evaluating the relevant terms in the supergravity action such as $\int H^{2}$. The correction to the Bianchi identity $\left(\underline{2} . \overline{9}_{1}^{\prime}\right)$ will modify $H$ and therefore modify the evaluation of the metric coming from this term; this correction is also related by supersymmetry to additional terms in the action, which will likewise enter in computing explicitly the metric. Taking all these effects into account, one could in principle generate a string perturbation expansion which must add up to (2.1. $\left.\overline{3}_{1}^{\prime}\right)$, since it is determined by the symmetries, the hyper-Kähler structure, and the one-loop effect that determines the topology. 


\subsection{Exact metric}

In determining the metric to all finite orders in $\alpha^{\prime}$, we have used a symmetry under shifts of the $B$-field period. This symmetry is violated by worldsheet instantons wrapped on the exceptional divisor $S$. Moreover [i $\left[\begin{array}{l}1 \\ -1\end{array}\right.$, such instantons do correct the metric on $\mathcal{M}$, because there are no worldsheet fermion zero modes except the minimal set required by supersymmetry. (In fact, the normal bundle to $S$ is $\mathcal{O}(-2)$, and the gauge bundle is trivial; eq. (3.2) of [1] 1 - $]$ is thus applicable and shows that the instanton contribution is not zero.) The instanton contributions vanish exponentially fast at infinity on $\mathcal{M}$ (since they are proportional to $\exp \left(-A / 2 \pi \alpha^{\prime}\right)$ with $A$ the area of $S)$. So we want a hyper-Kähler metric that has $\mathrm{SU}(2)_{R}$ symmetry, rotating the complex structures, and is exponentially close to (2.1. $\left.\overline{3}_{1}^{\prime}\right)$ at infinity.

Four-dimensional hyper-Kähler metrics with such an $\mathrm{SU}(2)_{R}$ symmetry and no $\mathrm{U}(1)_{A}$ symmetry have been classified in [i] 1 i and only if the first Chern class at infinity is -4 or -2 (or +4 or +2 if one takes the opposite orientation on the fiber, which corresponds to starting with the opposite hyper-Kähler structure on $\left.\left(\mathbb{R}^{3} \times S^{1}\right) / \mathbb{Z}_{2}\right)$. The smooth manifold with first Chern class -4 is often called the Atiyah-Hitchin space $\mathcal{M}_{A H}$; it is the moduli space of BPS dimonopoles on $\mathbb{R}^{3}$. The fundamental group of $\mathcal{M}_{A H}$ is $\mathbb{Z}_{2}$; it therefore has a double cover, which is the smooth manifold with first Chern class at infinity -2 .

The fact that the first Chern class at infinity that we computed (namely -4) corresponds to one of the values leading to a smooth manifold suggests that the moduli space we want is in fact $\mathcal{M}_{A H}$. Can we argue a priori that $\mathcal{M}$ should be smooth?

In the introduction, we argued that near an orbifold singularity without small instantons, the effective heterotic string coupling (if small at infinity) is uniformly small, so that nonperturbative effects should not arise. For example, this means that $\mathcal{M}$ should not have singularities interpreted in terms of nonperturbative massless particles or a non-trivial infrared CFT. Any singularity in $\mathcal{M}$ must make sense from the point of view of conformal field theory.

Conformal field theory corresponds to the tree approximation to string theory, so this means that any singularity in $\mathcal{M}$ should have an interpretation in the tree approximation to a weakly coupled classical field theory. Moreover (since we are looking at the heterotic string on a hyper-Kähler four-manifold), this must be a supersymmetric field theory in six dimensions. This is very restrictive: in this framework, the only mechanism to generate a singularity is via un-Higgsing of a gauge symmetry. For example, an orbifold singularity could be interpreted in classical field theory in terms of restoration of a discrete gauge symmetry. A $\mathbb{Z}_{2}$ orbifold singularity can likewise be interpreted in terms of un-Higgsing of a U(1) gauge symmetry as in the hyper-Kähler quotient considered in (2....... symmetry using a construction discussed in [i2 $\overline{6}]$. 
Thus, the orbifold singularities of the supergravity moduli space $\mathcal{M}_{\text {SUGRA }}=$ $\left(\mathbb{R}^{3} \times S^{1}\right) / \mathbb{Z}_{2}$ could be interpreted in classical supersymmetric field theory. String perturbation theory corrects this to a moduli space $\mathcal{M}_{\alpha^{\prime}}$ with a metric given in (2.13i). Now, instead of two $A_{1}$ singularities, there is a single $D_{4}$ singularity. Again, this could be interpreted in terms of classical field theory in terms of restoration of either a discrete gauge symmetry (the dihedral symmetry $D_{4}$ ) or a continuous gauge symmetry (the gauge symmetry used [23, 240 in interpreting the $D_{4}$ singularity as a hyper-Kähler quotient).

However, since the worldsheet instanton corrections to the metric are nonzero, this is not an option for the description of the moduli space. The conformal field theory moduli space $\mathcal{M}$ has the $\mathrm{SU}(2)_{R}$ symmetry with three-dimensional orbits, but no $\mathrm{U}(1)_{A}$ symmetry. We can now use the analysis in [1] $\overline{8}_{-1}^{1}$, chapter 9]. Hyper-Kähler metrics with the asymptotic behavior we want and no $\mathrm{U}(1)_{A}$ symmetry correspond to trajectories that flow to the point labeled $Q$ in diagram 4 of that chapter and do not lie on the line $Q B$. It is shown in [1] $\left.\overline{1}_{1}^{1}\right]$ that hyper-Kähler manifolds obtained this way are either $\mathcal{M}_{A H}$ or its double cover, or have a singularity in real codimension one where the trajectory originates at $B$. Such a real codimension one singularity cannot arise from a hyper-Kähler quotient, and so could not be interpreted in weakly coupled supersymmetric field theory. Given this, the arguments in the introduction plus the nonvanishing of the instanton corrections imply $\mathcal{M}=\mathcal{M}_{A H}$.

\subsection{Linear sigma model approach}

Smoothness of the moduli space $\mathcal{M}$ presumably means that the conformal field theory describing the heterotic string at an $A_{1}$ singularity, without small instantons, is uniformly valid for small string coupling constant. This contrasts with the case of a small instanton, where the effective string coupling diverges and nonperturbative phenomena occur no matter how weak the bare string coupling might be.

The arguments in the introduction suggest that more generally, a singularity in the metric with no singularity of the gauge field tends not to cause a breakdown of heterotic string perturbation theory. We will here give a simple linear sigma model argument that supports this expectation for a large class of examples. In making

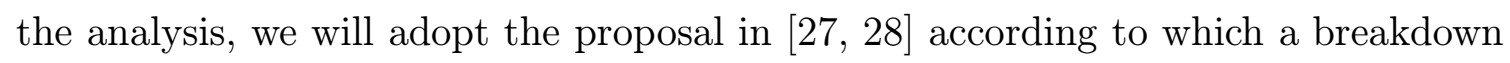
of conformal field theory should be detected by a failure of normalizability when the quantum states "spread" in a new direction in field space.

We consider the heterotic string on an $n$-dimensional complex hypersurface $K$ defined by an equation

$$
F\left(\phi_{1}, \phi_{2}, \ldots, \phi_{n+1}\right)=0
$$

in $n+1$ complex variables $\phi_{1}, \phi_{2}, \ldots, \phi_{n+1} . K$ is smooth if the equations

$$
0=F=\frac{\partial F}{\partial \phi^{i}}
$$


have no common solution. $K$ can be regarded as a noncompact Calabi-Yau manifold. We want to consider what happens when, by varying a parameter, a singularity develops. For example, we may take

$$
F=\sum_{i=1}^{n+1} \phi_{i}^{2}+\epsilon
$$

with a parameter $\epsilon$. In this case, the hypersurface $K$ develops a "conifold" singularity as $\epsilon \rightarrow 0$. In studying the perturbative heterotic string near a singularity in $K$, we will assume that the gauge bundle is trivial. This means that the left-moving gauge fermions of the heterotic string will be free fields, decoupled from the $(0,2)$ or $(0,4)$ sigma model that will describe the motion of strings on $K$.

Indeed, if $n>2$, the sigma model with target $K$ is a $(0,2)$ model, while for $n=2$, it is a $(0,4)$ model. Related to this, if $n>2$, the only moduli of such a singularity are the complex structure moduli that are present in $K$. For $n=2$, because there are collapsing two-spheres at the singularity, there are additional Kähler and $B$-field moduli.

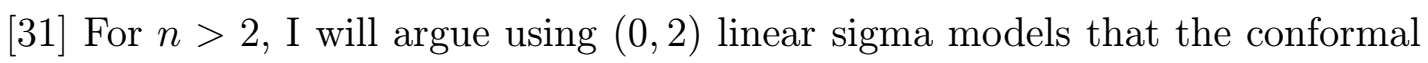
field theory remains nonsingular as $K$ develops a singularity. ${ }^{5}$ For $n=2$ (which is the case most directly relevant to the present paper) it is difficult to construct an equally satisfactory $(0,4)$ linear sigma model, and the argument based on $(0,2)$ linear sigma models is less satisfactory because it does not exhibit all of the moduli. But I believe the result is still true for $n=2 .{ }^{6}$

To construct a linear sigma model that should flow in the infrared to the $(0,2)$ superconformal field theory with target space $K$, we work in $(0,2)$ superspace (described more fully in $\left.\left[{ }_{3}^{3} 2\right]\right)$ with supercovariant derivatives $D_{+}, \bar{D}_{+}$obeying $D_{+}^{2}=$ $\bar{D}_{+}^{2}=0,\left\{D_{+}, \bar{D}_{+}\right\}=\partial_{+}$. To get $(0,2)$ supersymmetry, $D_{+}$and $\bar{D}_{+}$both have a spinor index of the same chirality, as do the fermionic coordinates $\theta^{+}, \bar{\theta}^{+}$of superspace. We introduce bosonic chiral superfields $\Phi_{1}, \ldots, \Phi_{n+1}$, obeying $\bar{D}_{+} \Phi_{i}=0$. They can be expanded $\Phi_{i}=\phi_{i}+i \theta^{+} \psi_{i}^{-}+\cdots$, with $\phi_{i}$ and $\psi_{i}^{-}$complex bosonic and fermionic fields, respectively; $\psi_{i}^{-}$is of definite chirality. The conventional kinetic

\footnotetext{
${ }^{5}$ Arguments along these lines have been developed in detail in [2 $\left.291,3{ }_{1}^{1}\right]$, and some aspects were explored in [3ij].

${ }^{6}(0,4)$ linear sigma models were constructed in [24] by considering $D 1$-brane probes of ADE singularities. Such models often have gauge anomalies, which were interpreted in [20 inflow to the probe from the bulk of spacetime; but this interpretation does not seem relevant for our consideration of heterotic string conformal field theory. An anomaly-free $(0,4)$ linear sigma model with exactly the properties we would want to exhibit all the moduli and establish our claim for $n=2$ does not seem to exist. An oversight in the original version of this paper (where an anomalous model was considered) was pointed out by M. Aganagic and A. Karch; I thank them and A. Mikhailov for discussions.
} 
energy for these fields is contained in the superspace expression

$$
L_{k i n}=\int d^{2} \sigma d^{2} \theta^{+} \sum_{i} \bar{\Phi}_{i} \partial_{-} \Phi_{i} .
$$

In addition, we introduce a fermionic chiral superfield $\Lambda^{+}=\lambda^{+}+\theta^{+} p+\cdots$, where $\lambda^{+}$ is a complex fermion of opposite chirality to $\psi_{i}^{-}$, and $p$ is a complex bosonic auxiliary field. The kinetic energy for $\lambda^{+}$, together with a $|p|^{2}$ term, comes from

$$
L_{a u x}=\int d^{2} \sigma d^{2} \theta^{+} \bar{\Lambda}^{+} \Lambda^{+} .
$$

These multiplets are coupled by a "superpotential" interaction

$$
L_{\text {super }}=\int d^{2} \sigma d \theta^{+} \Lambda^{+} F\left(\Phi_{i}\right)+\text { c.c. }
$$

Note that the integrand must be a chiral superfield in order for (2.19.1) to be supersymmetric; that is why $F$ must be holomorphic. We will assume moreover that $F$ is a polynomial, so that the superrenormalizable quantum field theory we are constructing exists rigorously, and the only issue is what it flows to in the infrared. After performing the $\theta$ integral, $L_{\text {super }}$ gives an interaction $p F\left(\phi_{i}\right)$ (plus a Yukawa coupling that gives mass to $\lambda^{+}$together with a $\phi$-dependent linear combination of the $\psi_{i}^{-}$). After integrating out the auxiliary field $p$ using the $|p|^{2}$ term from $L_{\text {aux }}$, we get then an ordinary potential

$$
V=\left|F\left(\phi_{i}\right)\right|^{2}
$$

The space of classical zeroes of $V$ is thus the hypersurface $K$ defined by $F=$ 0 . If $K$ is smooth, this model presumably flows to a $(0,2)$ superconformal field theory with that target. Even if $K$ is not smooth, as long as its singularities are isolated, the possible occurrence of a singularity in $K$ should not affect the welldefinedness of this conformal field theory. For example, in this theory, since $F$ (being a polynomial) grows if one is far from $K$, the wave functions decay rapidly when far away from $K$, whether $K$ is singular or not. The only unnormalizability of the quantum states comes from the noncompactness of $K$, and assuming the singular set of $K$ is compact (by holomorphy this is so precisely if the singularities of $K$ are isolated) this unnormalizability is not affected by the singularities. When $K$ develops a singularity, the conformal field theory becomes strongly coupled and difficult to analyze near the singularity, but nonetheless should continue to be well-behaved.

$(2,2)$ models. Since the above arguments may appear to be based on almost nothing, let us now show that in fact a similar analysis with a singularity in the gauge bundle as well as in the geometry gives a different result. We will consider the special case of "embedding the spin connection in the gauge group," which ensures that the gauge bundle becomes singular when the geometry does. To study this case, we 
must consider $(2,2)$ superconformal field theories, and formulate our linear sigma

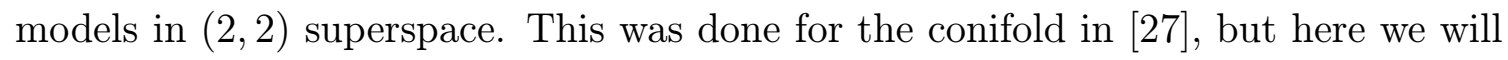
follow a more elementary and direct route. We introduce bosonic chiral superfields $\Phi_{i}=\phi_{i}+\cdots$ and $\mathcal{P}=P+\cdots$ and a superpotential

$$
W=\mathcal{P} F\left(\Phi_{i}\right)
$$

The ordinary potential is as usual in $(2,2)$ models $|d W|^{2}$, which in this case gives

$$
V=|F|^{2}+|P|^{2} \sum_{i}\left|\frac{\partial F}{\partial \phi_{i}}\right|^{2} .
$$

A classical zero of $V$ with $P \neq 0$ must have $F=d F=0$. Hence, if the hypersurface $K$ is smooth, all zero energy states have $P=0$. The space of such states is the hypersurface $K$ obtained by setting $F=0$ in $\mathbb{C}^{n+1}$. Thus, for smooth $K$, the model should flow in the infrared to a sigma model with target space $K$. So far this discussion is rather like the $(0,2)$ case. But now suppose that $K$ is singular. Setting the $\phi_{i}$ to a singular point of $K$, that is a solution of $\left(2 . \overline{1} \overline{5}_{1}^{\prime}\right)$, we now get a new branch of the moduli space of vacua by taking $P \neq 0$. The new branch is not compact, since $P$ can be arbitrarily big. The ability of quantum states to spread on this new branch should be expected to lead to a breakdown of the conformal field theory. Indeed, in the case of the conifold (for $n=3$ ), the familiar pole in Yukawa couplings has been computed [3]1] from the "leaking" of quantum states onto the new branch. This computation was actually done in a linear sigma model realization of the conifold different from what we have given above; the fact that different linear sigma model formulations show the occurrence of a new branch at the singularity encourages us to believe that this is an intrinsic phenomenon of the singularity and not an artifact of a particular linear sigma model formulation.

Comparison with bundle singularities. The opposite of the situation we have just looked at is a singularity in the gauge bundle on a smooth manifold. It has been argued [3]1; section 5.1] that in a large class of linear sigma models, a gauge singularity on a smooth manifold does result in a breakdown of conformal field theory. For a particular case (in complex dimension three) a proposal has been made concerning the nature of the resulting nonperturbative physics [3]3]. These results generalize the small instanton story for $n=2$. When conformal field theory does break down because of a singularity, the nature of the breakdown is not fully understood. In the

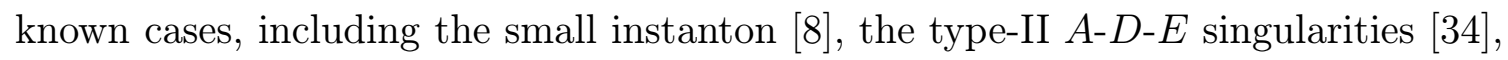

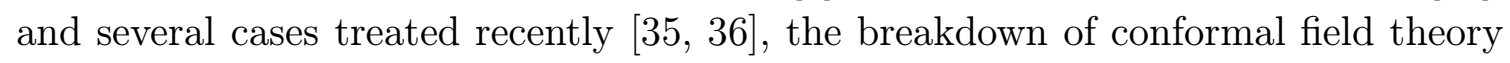
can apparently be described by the appearance, after suitable change of variables, of a linear dilaton field with a blowup of the string coupling constant at one end. A framework for understanding this has been proposed [3글.]. 


\section{A. Structure of $(0,2)$ moduli space}

Let $Y$ be a Calabi-Yau threefold; keep its complex structure fixed in this discussion. The remaining moduli of a $(0,2)$ supersymmetric model with target $Y$ are the gauge bundle moduli and the Kähler moduli. The definition of a holomorphic vector bundle depends only on the complex structure and not the Kähler metric. One might therefore think that in the supergravity approximation, the $(0,2)$ moduli space would be a product $\mathcal{M}_{G} \times \mathcal{M}_{K}$ of a gauge bundle moduli space $\mathcal{M}_{G}$ and a Kähler moduli space $\mathcal{M}_{K}$. This was assumed in [3] ${ }_{-}$, , section 5.2], but is inaccurate for several reasons. (The inaccuracy has been corrected in the hep-th version of the paper.) One reason is that $\mathcal{M}_{G}$ is the moduli space of stable bundles, and the condition for stability "jumps" as the Kähler metric varies. As a result, $\mathcal{M}_{G}$ undergoes birational transformations as one moves about in $\mathcal{M}_{K}$, a phenomenon explored in [3-īi]. There is a reciprocal effect which, by itself, would cause $\mathcal{M}_{K}$ to be fibered over $\mathcal{M}_{G}$. Indeed, given that the $B$-field periods are part of the Kähler multiplets, a non-trivial fibration of $\mathcal{M}_{K}$ over $\mathcal{M}_{G}$ follows from the fact developed in [1] $\left.{ }_{1}^{1} \overline{\sigma_{i}}\right]$ and used in section ${ }_{2}^{-} . \overline{2}_{1}^{\prime}$ above: the $B$-field periods take values in a circle bundle, not just in U(1). (The nontrivial fibration of the $B$-field periods over $\mathcal{M}_{G}$ comes from the $\operatorname{tr} F \wedge F$ term in the Bianchi identity for $B$; this term is present in the minimal supergravity.)

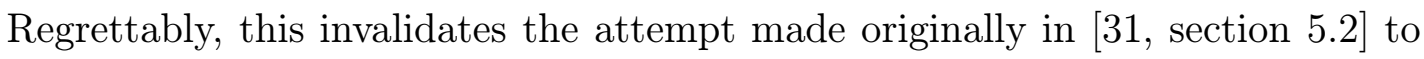
argue conformal invariance of $(0,2)$ models directly from nonlinear sigma models. (The rest of the paper is based on quite different arguments using linear sigma models.) Note that the formula for the instanton contribution to the superpotential given in [i] $\overline{6}$ ] varies holomorphically with the parameters, showing that, because of the nontrivial fibration, it is possible for the contribution of a given instanton to make a nonvanishing contribution to the superpotential that obeys all conditions of holomorphy.

\section{Acknowledgments}

I would like to thank H. Ooguri for helpful comments.

This work was supported in part by NSF Grant PHY-9513835 and by the Caltech Discovery Fund.

\section{References}

[1] B. de Wit, P. Lauwers and A. Van Proeyen, Lagrangeans of $N=2$ supergravity-matter

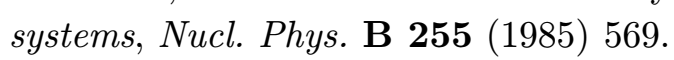

[2] S. Kachru and C. Vafa, Exact results for $N=2$ compactifications of heterotic strings,

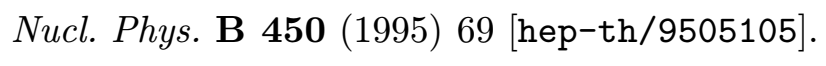

[3] S. Ferrara, J.A. Harvey, A. Strominger and C. Vafa, Second quantized mirror symme-

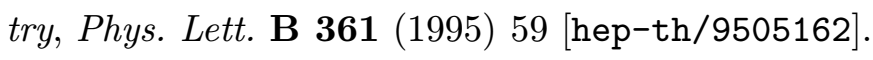


[4] S. Kachru, A. Klemm, W. Lerche, P. Mayr and C. Vafa, Nonperturbative results on

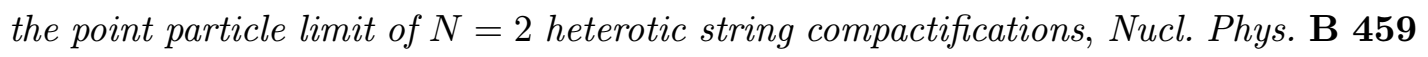
:

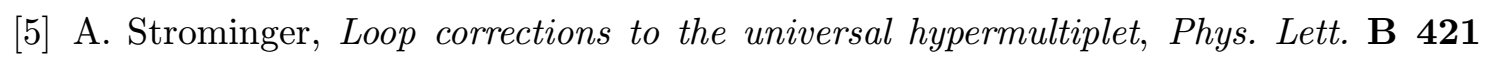
: -

[6] P.S. Aspinwall, Aspects of the hypermultiplet moduli space in string duality, 'IJ. $\bar{H}_{-}^{-} \overline{i g h}$

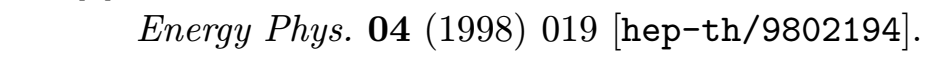

[7] A. Strominger, Massless black holes and conifolds in string theory, i -

[8] C.G. Callan Jr., J.A. Harvey and A. Strominger, World sheet approach to heterotic instantons and solitons, Nucl. Phys. B $\mathbf{3 5 9}(1991) 611$

[9] E. Witten, Small instantons in string theory, [hep-th/9511030i,

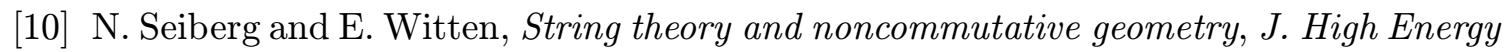
[.

[11] P.S. Aspinwall and D.R. Morrison, Point-like instantons on K3 orbifolds, iNuchel. Phys.: !

[12] K. Intriligator, $R g$ fixed points in six dimensions via branes at orbifold singularities, iNucl. Phys B 496 (1997) 177i [hep-th/9702038'.

[13] J.D. Blum and K. Intriligator, Consistency conditions for branes at orbifold singular-

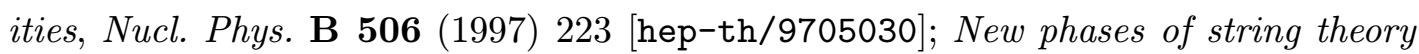

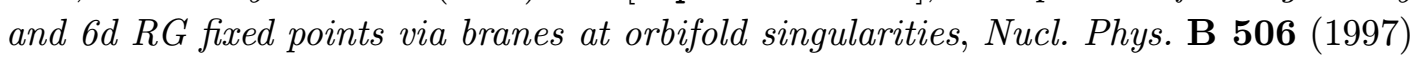
199.

[14] P.S. Aspinwall and R.Y. Donagi, The heterotic string, the tangent bundle and derived categories, 'Adv. Theor Math. Phys. 2 (1998) 1041i [hep-th/9806094'].

[15] P. Berglund and P. Mayr, Heterotic string/F-theory duality from mirror symmetry, Adv. Theor. Math. Phys. 2 (1999) 1307i hep-th/9811217il.

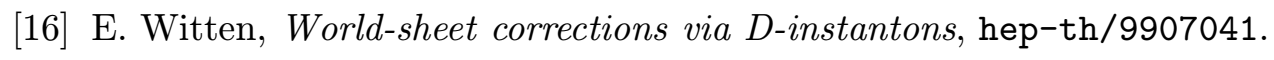

[17] G.W. Gibbons and C.N. Pope, The positive action conjecture and asymptotically eu-

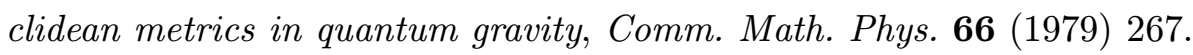

[18] M. F. Atiyah and N. Hitchin, The geometry and dynamics of magnetic monopoles, Princeton University Press, Princeton 1988.

[19] N. Seiberg and E. Witten, Gauge dynamics and compactification to three dimensions, hep-th/9607163.

[20] A. Sen, Dynamics of multiple Kaluza-Klein monopoles in $M$ - and string theory, ${ }^{i} \bar{A} \bar{d} \overline{-} \bar{v}^{-}$ . 


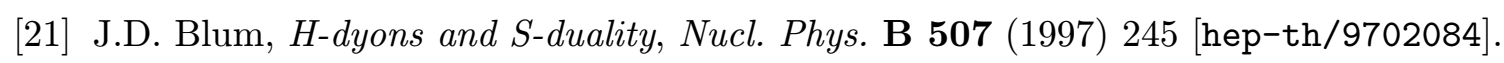

[22] T. Eguchi and A.J. Hanson, Asymptotically flat selfdual solutions to euclidean gravity, 'P $\bar{h} \bar{y}$ s.

[23] P. Kronheimer, The construction of ALE spaces as hyper-Kähler quotients, J. Diff. Geom. 28 (1989) 665.

[24] M.R. Douglas and G. Moore, D-branes, quivers and ALE instantons, hep-th/960316

[25] G.W. Gibbons and P. Rychenkova, Hyper-Kähler quotient construction of BPS monopole moduli spaces, hep-th/9608085:

[26] N. Seiberg and E. Witten, Monopoles, duality and chiral symmetry breaking in $N=2$ supersymmetric QCD, iNucl. Phys. B 431 19994$)$ 484, [hep-th/9408099'].

[27] E. Witten, Phases of $N=2$ theories in two dimensions, 슨 1.

[28] E. Witten, Some comments on string dynamics, in Strings '95: future perspectives in string theory, I. Bars et al. eds., [hep-th/95071211].

[29] J. Distler, B.R. Greene and D.R. Morrison, Resolving singularities in (0,2) models, iNucl. Phys. B 481 (1996) 289: [hep-th/9605222].

[30] T.-M. Chiang, J. Distler and B.R. Greene, Some features of (0,2) moduli space, Nㅡㄴ :

[31] E. Silverstein and E. Witten, Criteria for conformal invariance of (0,2) models, iNu

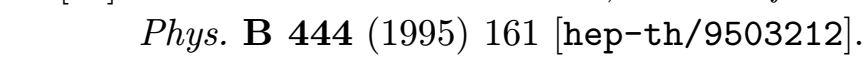

[32] C.M. Hull and E. Witten, Supersymmetric sigma models and the heterotic string, 'Ph $\underline{\text { Phys.' }}$ L

[33] S. Kachru, N. Seiberg and E. Silverstein, Susy gauge dynamics and singularities of $4 D$ $N=1$ string vacua, iNucl. Phys. B 480 (1996) 170 [iep-th/9605036i.

[34] H. Ooguri and C. Vafa, Two-dimensional black hole and singularities of CY manifolds,

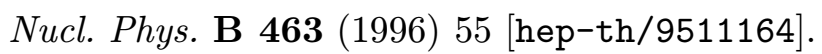

[35] N. Seiberg and E. Witten, The D1/D5 system and singular CFT, 'J. High Energy (1)

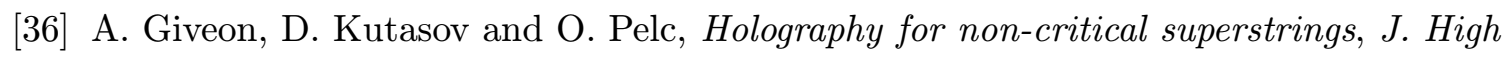

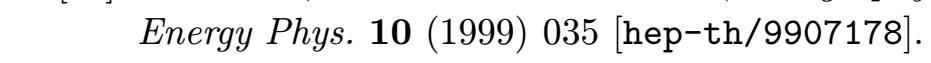

[37] O. Aharony and M. Berkooz, IR dynamics of $D=2, N=(4,4)$ gauge theories and DLCQ of 'little string theories', 'J. High Energy Phys. 10 (1999) 030', [rep-th'-

[38] E. Sharpe, Kähler cone substructure, Ä dv [hep-th/9810064. 